\title{
Domain Experts on Dementia-Care Technologies: Mitigating Risk in Design and Implementation
}

\author{
Clara Berridge $^{1}\left(\right.$ D $\cdot$ George Demiris $^{2} \cdot$ Jeffrey Kaye ${ }^{3}$
}

Received: 12 May 2020 / Accepted: 21 December 2020 / Published online: 18 February 2021

(c) The Author(s) 2021, corrected publication 2023

\begin{abstract}
There is an urgent need to learn how to appropriately integrate technologies into dementia care. The aims of this Delphi study were to project which technologies will be most prevalent in dementia care in five years, articulate potential benefits and risks, and identify specific options to mitigate risks. Participants were also asked to identify technologies that are most likely to cause value tensions and thus most warrant a conversation with an older person with mild dementia when families are deciding about their use. Twenty-one interdisciplinary domain experts from academia and industry in aging and technology in the U.S. and Canada participated in a two-round online survey using the Delphi approach with an $84 \%$ response rate and no attrition between rounds. Rankings were analyzed using frequency counts and written-in responses were thematically analyzed. Twelve technology categories were identified along with a detailed list of risks and benefits for each. Suggestions to mitigate the most commonly raised risks are categorized as follows: intervene during design, make specific technical choices, build in choice and control, require data transparency, place restrictions on data use and ensure security, enable informed consent, and proactively educate users. This study provides information that is needed to navigate person-centered technology use in dementia care. The specific recommendations participants offered are relevant to designers, clinicians, researchers, ethicists, and policy makers and require proactive engagement from design through implementation.
\end{abstract}

Keywords Dementia $\cdot$ Alzheimer's disease $\cdot$ Long-term care $\cdot$ Ethics $\cdot$ Decision making

Clara Berridge

clarawb@uw.edu

Extended author information available on the last page of the article 


\section{Introduction}

Investment in technology to support dementia care is growing as demand for human and financial resources to support care outpaces its supply (AARP and National Alliance for Caregiving 2020). The functions of technological tools range widely, including activities of daily living (ADL) assistance, behavioral and health monitoring, cognitive assistance and monitoring, and environmental and emotional support (Choi et al. 2019; Seelye et al. 2020; Piau et al. 2019; Czaja et al. 2019; Orlov 2019). While ethical implications of the use of technologies for people living with dementia have been slow to come into focus (Ienca et al. 2018; Robillard et al. 2018; Novitzky et al. 2015), issues such as autonomy, informed consent with dementia, dignity, and distributive justice, along with threats to values like privacy and identity, are beginning to receive heightened attention in the literature (Meiland et al. 2017; Robillard et al. 2018; Mulvenna et al. 2017; Sánchez et al. 2017; Berridge 2016; Boise et al. 2013). Researchers have identified adoption barriers and user dissatisfaction that result when ethics and implicated values are not engaged in the development of devices (Robillard et al. 2018). The surveilling nature of some of these technologies can also put important values - and family members themselves-in tension with one another (Kenner 2008; Berridge and Wetle 2019).

An expert study that probed some of the pressing issues with Internet-connected technologies in dementia care was recently conducted in Italy, Switzerland, and Germany (Wangmo et al. 2019). Twenty medical workers, nursing home managers, and researchers in geriatrics and related fields were asked about ethical concerns with the use of intelligent assistive technology with older adults and people living with dementia. A range of concerns were described, including fair technology access, the possible replacement of human assistance, and the use of deception. The ethical importance of communicating risks and benefits to users of a given technology and consenting patients were core concerns (Wangmo et al. 2019). These concerns are especially amplified in this period of rapid technological development, with marked growth of innovations in robotics, artificial intelligence (AI), sensor-based systems (Vallor 2016; Lindeman et al. 2020), including in-home sensors that monitor movement and behavior, and voice-activated systems that access information or remotely control appliances. A recent systematic review found that most $(67 \%)$ dementia care technologies have been developed without explicit consideration of any ethical principles (Ienca et al. 2018).

What information is needed to prepare to navigate new technology-based practices in home-based dementia care in an way that respects the values that matter to individuals and families? One need is to be able to project as best we can what technologies to focus on. Another need-underscored by the ethics research-is a clear understanding of their potential risks and benefits. While big-picture ethical considerations must come into sharper focus (including questions about access, autonomy, and what constitutes good care), we must also examine the specificity of individual technologies and their attendant uses and risks (Pols 2017).

This study attends to these needs by consulting transdisciplinary aging and technology experts from academia and industry. The goal is threefold: to learn 
which technologies are predicted to be the most prevalent in five years; to understand the potential benefits and risks of each technology; and to identify options to mitigate those risks. Our hope is that these findings will allow for a concrete assessment of ways to address the risks and value tensions that technologies used by families in the home create. For this applied approach, we focus on identifying and learning about those technologies that are the most likely to be threatening to values that older adults may care about (i.e. privacy, independence) and thus most warrant a conversation with an older patient with mild dementia when families are deciding about their use. The overarching aim is to learn where to focus efforts for the near-term and to proactively foster technology-mediated practices in the home that families can feel good about.

\section{Methods}

\section{Participants and Recruitment}

Potential participants were identified in two ways. First, the three authors who are disciplinarily dispersed within the field of gerontechnology collectively identified an initial list of experts to invite. We sought a balanced mix of people with specific expertise in technology for older adults from industry, AI, and gerontology and related social sciences, including ethics. Second, some of these industry contacts and leaders in gerontechnology-including nonparticipants-were asked to help identify potential participants and those with specialized knowledge in under-represented areas of AI or ethics. Contacts were also asked if they could recommend others to invite from their own respective fields. The combined process yielded a list of 37 potential participants.

Participants were domain experts in aging and technology research, design, or implementation in the U.S. and Canada who had been in the field for more than five years. A criterion for academics was that they had published at least five peerreviewed articles on technology and aging; those in industry had recent work experience with technology products for elder care.

The sample size for the Delphi approach we pursued is not a statistically bound decision (Akins et al. 2005; Ziglio 1996), and 15-20 respondents is the typical range (Hsu and Sandford 2007). A pre-Delphi invitation to participate in the process was first sent to the 37 domain experts (Sinha et al. 2011). Each received one email invitation, and those with away messages received two. Participants were informed that this study would be the first step of a larger project of developing a new communication and education tool for early-stage dementia family-care partners to discuss how technologies may be used in their care. The role of this Delphi process in the development of the tool was to help determine which technologies should be featured in the tool in order to make it as relevant over time as possible.

Twenty-five responded that they were willing to participate in the study; this rate of agreement to participate of $67.5 \%$ was expected based on the Delphi literature (Mokkink et al. 2010). These 25 received the Round 1 survey and 21 of them completed it. There was no attrition between the two rounds: 21 participants completed 
Round 1, and all 21 completed Round 2, for an 84\% response rate (21/25). Of these participants, 15 (71\%) worked primarily as academics, many of whom had experience developing technology; and 5 (24\%) represented industry. One (5\%) was a consultant and industry analyst in the aging and technology space. Participants are listed in the Appendix.

\section{The Delphi Process and Analysis}

The Delphi technique is used to solicit experts' opinions and to build consensus (Hsu and Sandford 2007). To begin this process, a Round 1 structured questionnaire was distributed with nine questions. Participants were presented with nine technologies (e.g., virtual reality) and data types that monitoring technologies capture (e.g., location outside of the home) based on an extensive review of the literature. Since device forms will change more rapidly than will the types of data collected, the focus was on generic technologies or data types rather than specific products. Participants were asked to describe the primary benefits and risks for each for use in the care of a person with mild dementia. After listing the potential risks and benefits for each provided technology, participants were asked to expand and comment on the technology categories. Beginning a two-round survey with a Round 1 structured questionnaire is an acceptable modification of the three-round Delphi approach that begins with an open-ended questionnaire (Hsu and Sandford 2007). The Delphi method requires a minimum of two rounds (three if round one is open-ended) (Walker and Selfe 1996). As Walker and Selfe point out "repeated rounds may lead to fatigue by respondents and increased attrition" (p. 679). Considering the challenge of attrition for our expert participants, we decided to pursue a two-round approach.

For Round 2, twelve technology categories were rearticulated based on the most commonly reported beneficial uses for each and suggestions for missing categories from Round 1. Based on Round 1 responses, descriptions of technology purposes were refined, one category was subdivided into two, and two new technology categories were added: augmented reality and smart homes for environmental control.

The refined technology descriptions were presented for participants to rank according to those that they perceived would be most commonly used in the care of people with dementia in five years. Participants were then asked to select among the twelve technologies the ones that most warranted a conversation between an older person with dementia and the family members who were deciding about using them. Participants were prompted to consider which were the most likely to be threatening to values. Each question was followed by a prompt for participants to describe their rationale for their selection. Ranking and selecting questions had answer choices that were shuffled for each participant.

The most commonly noted risks generated in Round 1 were extracted and reported back to participants in aggregate in Round 2. Participant responses were anonymous to other participants. This report-back was followed by a question to identify design options that families might choose to mitigate the risks of using technologies in care. Participants were asked to describe realistic design options to mitigate as many of the noted risks as they could think of. 
Rankings were analyzed using frequency counts. Written-in responses were thematically analyzed using an inductive, reflexive thematic analysis approach by the first author and then validated by another member of the team (Clarke et al. 2019). Participants were compensated $\$ 300$ for completion of the two surveys. This research was approved by the University of Washington Human Subjects Division.

\section{Results}

\section{Near-Future Predicted Use}

After participants commented on the selection of technologies in Round 1, they were presented in Round 2 with a refined list of 12 technology categories, which are presented in Table 1.

They were then asked to rank according to those that would be the most prevalent in dementia care in five years and to explain their rankings. Prediction proved difficult. While the technologies that would be most common were ranked fairly consistently, there was no agreement about how near they were to widespread use. Projections varied widely, from "Some will be obsolete by five years" to "I believe all of the above will be very prevalent" and "I think widespread use of all these are more than five years away-more like ten." One participant explained, "We generally over estimate what is possible in two years and underestimate what is possible in five years. In many of these examples, we are assessing technical capacity (which is high in 5 years) with willingness to adopt (which I think is low)." Others observed that the gap between cost and income of those caring for older adults will limit uptake.

Table 1 Twelve refined categories of technologies used in dementia care

Smart home systems to control environmental settings and appliances

Augmented reality to give the person with dementia digital cues to support home environment and task navigation

Virtual reality for cognitive stimulation, relaxation, reminiscence therapy, behavior management

Location tracking within the home to detect variation in patterns of activity

Location tracking outside of the home to monitor safety and social activity

Detecting movements through apartment door to confirm return home and monitor outdoor activity frequency or presence of workers, meal delivery, visitors

Video conferencing that allows a caregiver to turn the webcam on and "enter" the room visually for social connection and visual assessment of person and home

Remotely monitoring physiological variables for frequent assessment to predict and manage healthrelated risks

Recording audio in a person's home to respond to emergencies and security threats, monitor socialization, or detect cognitive change and other conditions

Using an AI conversational agent to stimulate engagement and connection with the outside world (accessing music, information, web-conferencing with people)

Using an AI virtual agent or socially assistive robot for non-human companionship to ease loneliness and prompt cognitive or physical engagement

Using a robotic assistant to help with physical aspects of daily living 
Without reaching consensus about realistic timelines, participants on the whole believed that the following would be the most prevalent in five years (most to least frequently endorsed):

1. Smart home systems to control environmental settings and appliances

2. Video conferencing that allows a caregiver to turn the webcam on and "enter" the room visually for social connection and visual assessment of person and home

3. Location tracking outside of the home to monitor safety and social activity

4. Location tracking within the home to detect variation in patterns of activity

5. Detecting movements through apartment door to confirm return home and monitor outdoor activity frequency or presence of workers, meal delivery, visitors

6. Remotely monitoring physiological variables for frequent assessment to predict and manage health-related risks

7. Using an AI conversational agent to stimulate engagement and connection with the outside world (accessing music, information, web-conferencing with people)

8. Recording audio in a person's home to respond to emergencies and security threats, monitor socialization, or detect cognitive change and other conditions

9. Using an AI virtual agent or socially assistive robot for non-human companionship to ease loneliness and prompt cognitive or physical engagement

Key insights were that those technologies that already exist for a wider market, such as free GPS software on phones, smart home systems, and activity monitors, were predicted to be more prevalent sooner than those specifically targeting older adults. Those that are becoming ubiquitous were poised to be readily adopted for care purposes. With the growing ubiquity of smart speakers also comes greater attention to failures of data security, as one participant suggested: "Audio recording will be delayed in the consumer market, in part due to the strongly negative reactions we're seeing to things like smart speakers recording audio (e.g., bad reactions to audio recording in Amazon's Echo and Google's Home products)."

\section{Technology Agnostic Potential Benefits and Risks}

When the potential benefits and risks associated with each technology were identified (see Table 2), some of these were found to be technology independent, common across most of these individual technology categories. Concern over the security of audio and other data types was one of these common issues. Participants emphasized both security risks and nefarious use as unresolved problems. The issues were raised of data exfiltration by hackers or Internet service providers and "Big data collection needed without much knowledge for where it may end up." Much of the data collected by these technologies can be accessed by third parties, which leads to privacy risks for anyone whose data might be captured, including visitors to a home where the technology is in use. Participants voiced concern that recordings that lead to knowledge of non-adherence or high-risk activities could in turn lead to increased premiums or denial of healthcare coverage. 


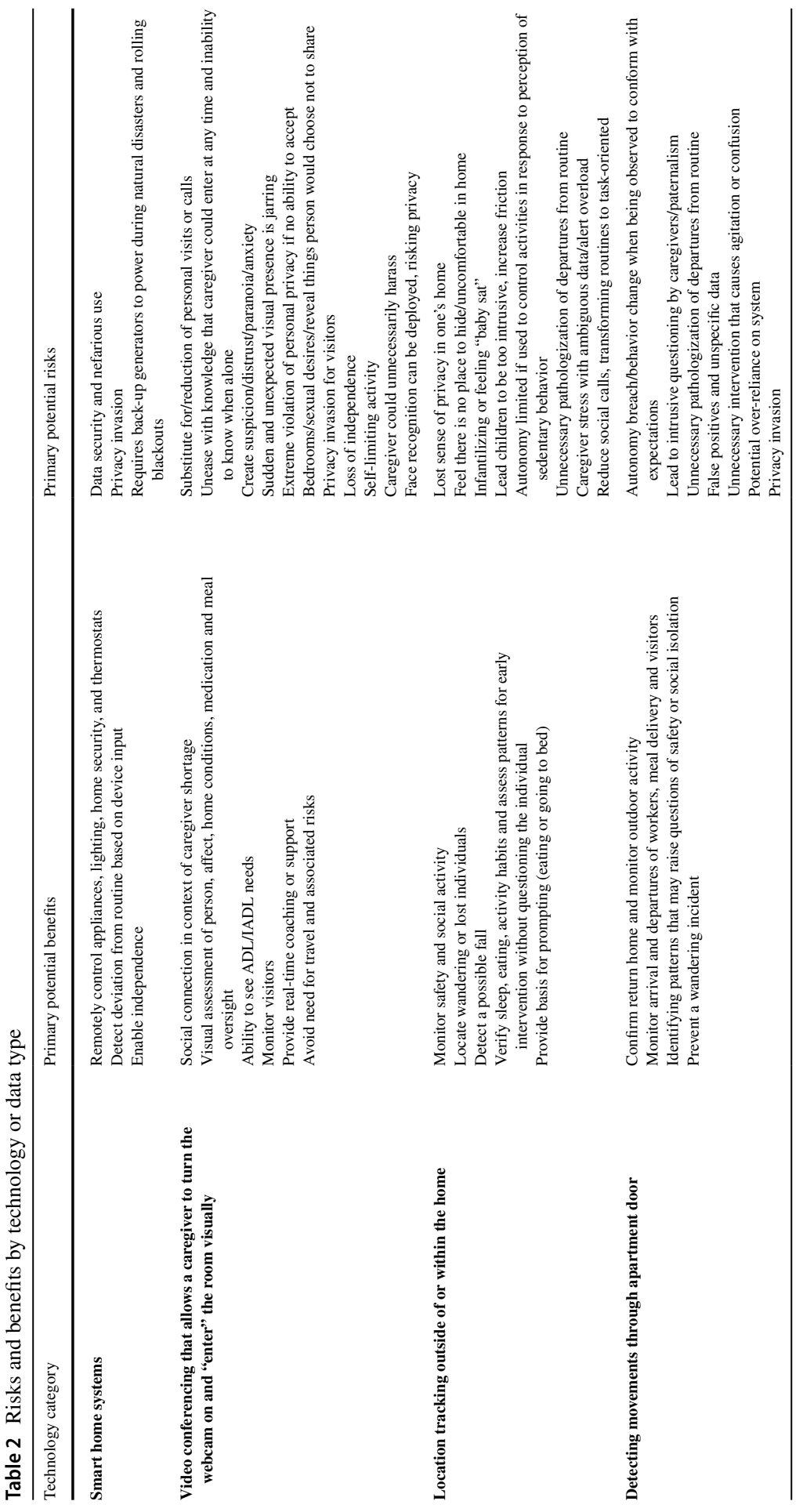




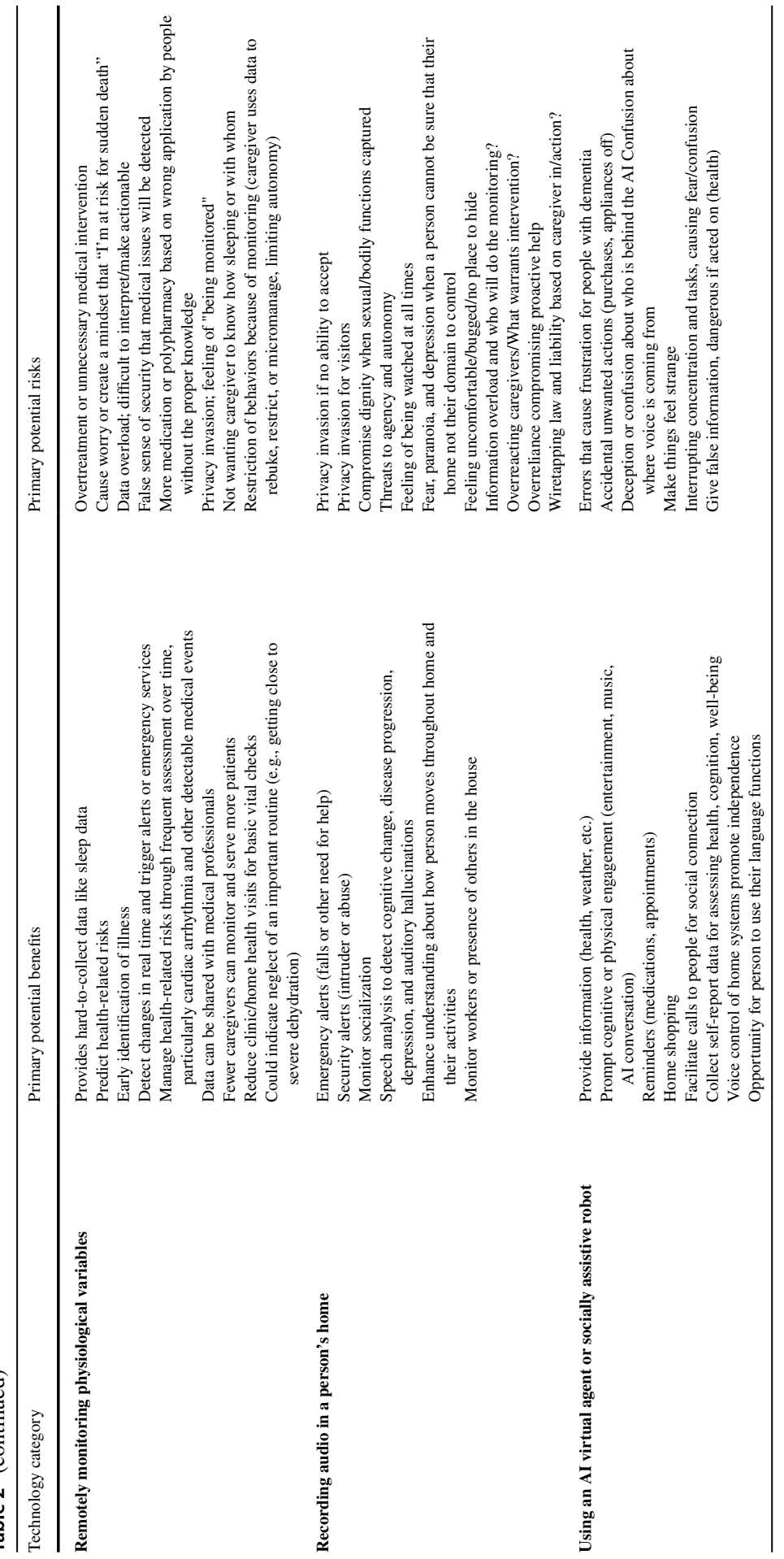




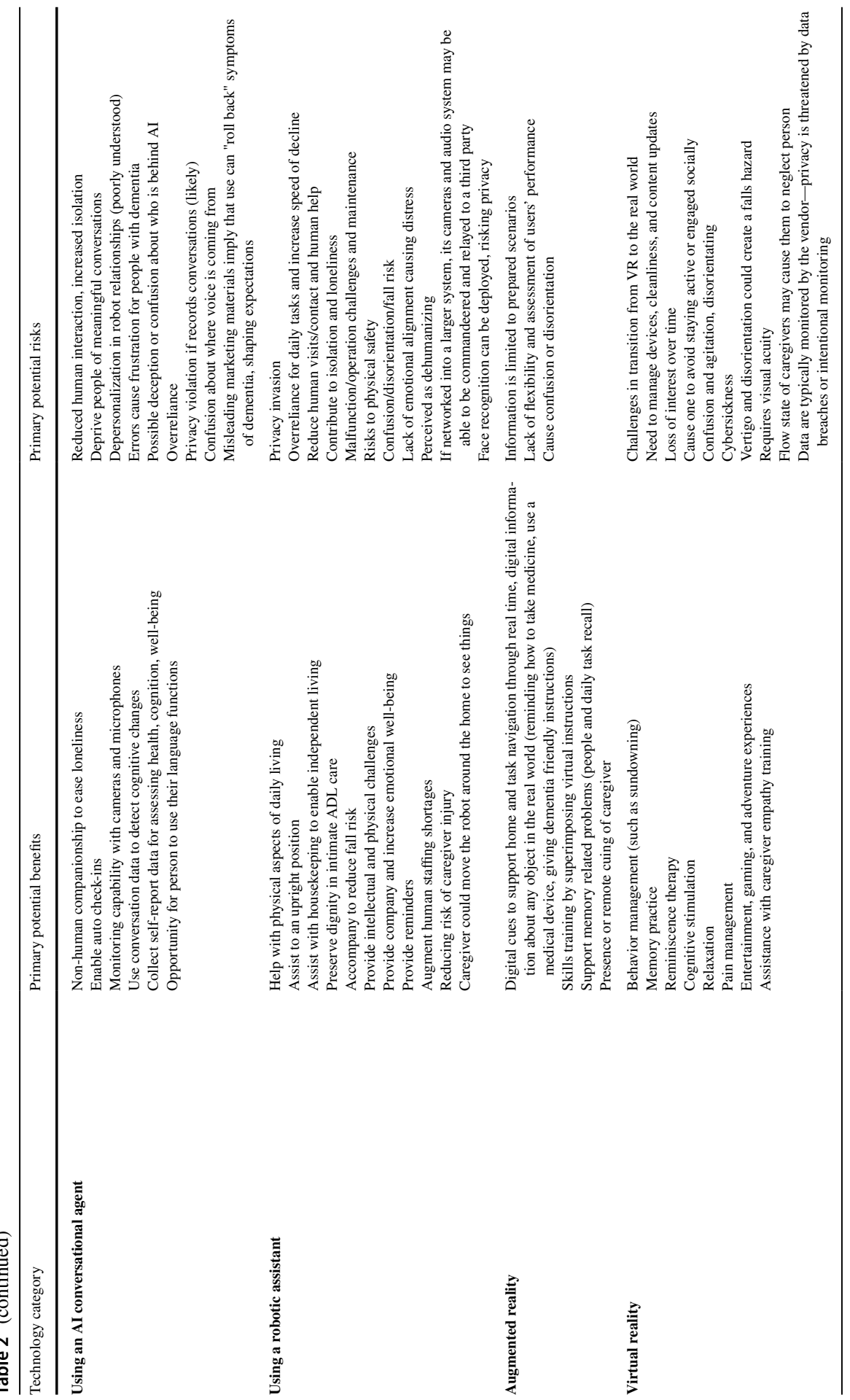


Prolonging independent living and caregiver peace of mind were seen as potential benefits across all technologies, while unactionable data, information or alert overload, and caregiver fatigue were cited as likely to make caregivers anxious. Other commonalities across technologies included risks of inaccurate data, false positives and negatives, and a false sense of safety. Finally, the problem that all Internet-connected devices require access to the Internet was identified as creating an impediment to use.

Other potential benefits and risks are specific to a given technology. The summarized potential benefits and risks of each individual technology is provided in Table 2.

\section{Mitigating Risks}

Participants were then presented with the aggregated risks most commonly noted in Round 1 (shown in Table 3) and asked in Round 2 to identify options to mitigate the risks of using these technologies in care.

Participants acknowledged the great difficulty of addressing these challenges. One wrote, "This is really hard, all the concerns are very valid." Another participant responded:

Table 3 Aggregated predominant risks of technology use

Privacy invasion for visitors and person living with dementia (reveal a state that an individual finds embarrassing or would choose not to share)

Infantilizing or feeling "baby sat"

Feeling uncomfortable/bugged/no place to hide, leading to fear, paranoia, depression

Pathologizing departures from routine

Caregiver stress from ambiguous data or alert overload

Reduction of personal visits or calls, leading to loneliness or isolation

Overtreatment or unnecessary intervention

False sense of security that issues will be detected, reducing proactive help

Tension caused by caregiver paternalism in form of intrusive/harassing questioning

Caregiver limits freedom, using data to rebuke, restrict, or micromanage

Care recipient changes behavior to conform with expectations

Unease with knowledge that caregiver could enter the room at any time and inability to know for sure when alone, creating suspicion/distrust/paranoia/anxiety

Over reliance on tech for daily tasks, speeding decline in functional capacity

Lack of emotional alignment causing distress

Compromising dignity (bodily functions and sexual activities captured)

Data lead to knowledge of non-adherence or high risk activities that lead to increased premiums or denial of coverage

Data security risk leading potentially to nefarious use

Unknown or unsanctioned third party use

Problems with errors, causing frustration for people with dementia

Person with dementia accidentally triggers unwanted actions

Possible deception or confusion about who is behind an AI agent or robot 
Wow this is a tough one. I believe that technology is best considered as a way to better enable or drive efficiencies with the in-person caregiving function rather than serve as a replacement for human touch/interaction. I feel that when considering the use of technology to enhance the care of adults with Alzheimer's or dementia it becomes even more critical to consider how the technology will primarily assist the caregiver and reduce their burden rather than be used as a substitute (which can contribute to so many of the unwanted outcomes identified in the above list) for their in-person engagement.

One participant explained the importance of getting implementation right:

The issue is not so much design options for the tech itself (although important), but ensuring that technology - whatever it is - is applied in the right way (e.g., care professionals have the right care process in place, including consent, services are appropriately regulated, tech outcomes are evaluated, consumers are sufficiently "educated" to understand the benefits and costs, etc.).

Suggestions given by participants for risk mitigation are outlined below and summarized in Table 4. They are categorized as follows: intervene during design; make specific technical choices; build in choice and control; place restrictions on data use and ensure security; require data transparency; enable informed consent; and proactively educate users.

\section{Intervene During Design}

Participants suggested three specific approaches that designers and engineers could use to inform development of dementia care technologies: value sensitive design, the privacy-by-design framework, and the ethical adoption framework. These recommended frameworks are described in the discussion section. Other suggestions that are compatible with these frameworks were to convene multidisciplinary design teams; to engage gerontechnologists with expertise in cognitive impairment, ethics, and product testing; and to conduct careful end-user testing, usability, and ethics studies prior to deployment. Ethics studies, which could be done quickly and inexpensively, were described as critical.

One particular design-stage ethical concern was raised repeatedly. Participants worried that some of the technologies, particularly those that are AI-driven, could be used to justify or enable reduction of human contact. One wrote, "Overall I continue to be very concerned about the ethics and invasiveness of some tech. AI and use in loneliness has little data and I worry about attempting to substitute real human connection." Another explained,

An AI for non-human companionship is problematic because it undermines perceived connection to family/care. Although family/care may not be any less, the introduction of the non-human companionship can be perceived as replacing family/care. AI to stimulate engagement does not have this same problem. It's about addressing boredom, encouraging mental stimulation, not about companionship. 
Table 4 Ways to mitigate risks

\section{Intervene during design}

Employ existing frameworks

Value sensitive design

Privacy-by-design framework

Ethical adoption framework

Convene multidisciplinary design teams with expertise in cognitive impairment, ethics, and product testing

Prior to deployment, conduct end-user and usability testing

Prior to deployment, conduct ethics studies

Do not design AI devices to substitute for human interaction

Avoid humanoid design and deception

\section{Technical choices}

Use non-identifying, privacy enhancing methods (sensor before camera; silhouette before image)

Uncouple data sources

Prohibit extraneous data collection

System check-in with individual before alert is triggered

Graded alerts

Auto-tag and remove activities compromising the person's dignity

Avoid continuous streaming—require permissions for video or audio

Enable two-way feed with caregiver

Titrate level of support to need to prevent skill loss

\section{Build in choice and control}

Enable ability for PWD to pause system/data collection

Enable data deletion and approval by individual

Employ end-user defined function metrics

Allow PWD to stop or not use

Provide alternative options to enable actual choice about use

\section{Restrictions for data security and unsanctioned third-party use}

Mandatory data security practices: two-factor authentication, encryption, HIPAA compliance, permissions

Individual or family owns their data

Disclosures that data won't result in higher health insurance costs

Ensure that enrollment is NOT through healthcare or insurance related benefit

Data should not be used for marketing

Require informed consent for use other than care provision, including research

Guaranteed assurances of data privacy

\section{Require data transparency}

Regarding: where data are, who can access, what are the algorithms to trigger intervention, possible data monetization

Devices actively remind users of how they operate, who is controlling them, and how data are being collected and used 
Table 4 (continued)

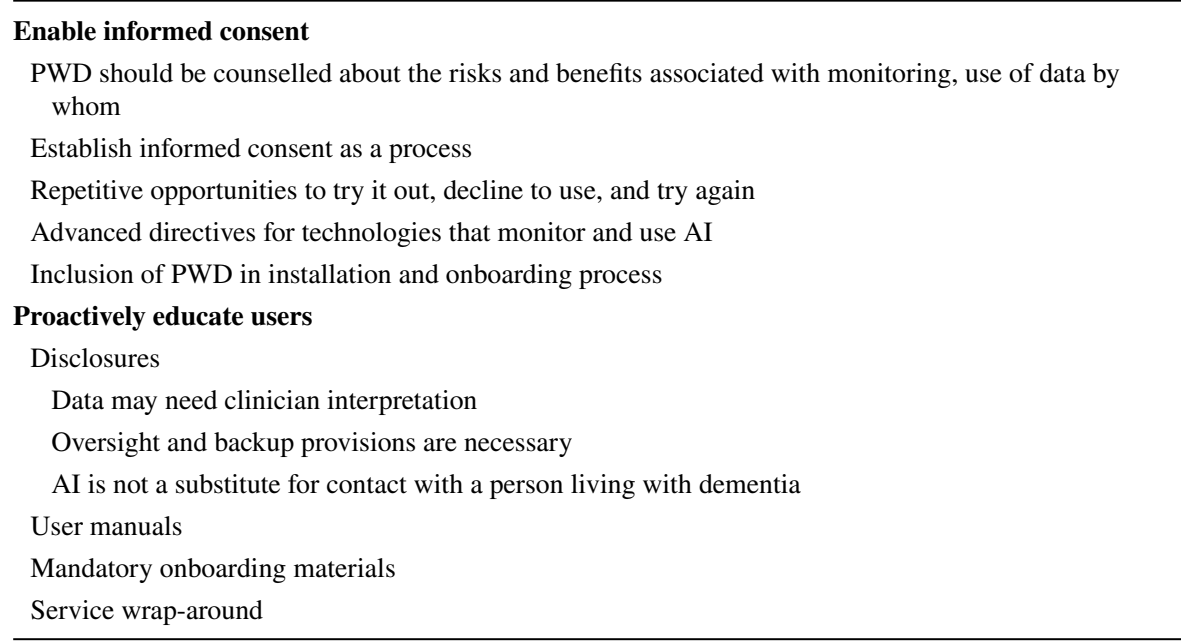

This was a common distinction made between appropriate and potentially inappropriate uses of AI. Some cautioned that substituting AI for human contact could also speed decline in functionality, emphasizing the importance of avoiding technology that "substitutes for maintaining personhood." Participants noted that humanoid design should be avoided to reduce the risk of deception around AI.

Participants also spoke to the difficulty of mitigating all the risks when much depends on the implementation of the technologies: "It's hard to control for the dynamics of interpersonal/family relationships. If a product is designed to do something that invades privacy and trades off that for safety, then there will always be this issue."

\section{Technical Choices}

Participants suggested specific technical options that could be designed in. One was that non-identifying methods should be used whenever possible. For example, while a camera could be used to detect falls, heat sensors or smart floors would preserve privacy more effectively. Participants wrote about the need to embed privacy preserving features in surveillance technologies, such as uncoupling data sources, capturing silhouettes rather than actual images, and collecting only necessary data while balancing between utility and data granularity.

To prevent triggering unwanted actions, participants suggested creating a system of checks and balances such that the system checks in with the person to gauge status before sending an alert. Alerts could also be graded to specify certainty that a negative event had occurred. Computer vision and machine-learning algorithms could be used to tag and remove activities that are likely to compromise the person's dignity. Participants suggested requiring permissions for intrusive applications, including 
video or audio, instead of continuous streaming. In an effort to equalize power relations between a person living with dementia and a caregiver, two-way feed could be provided when using audio or video capture.

To mitigate over-reliance on technology, systems could require people to exert some form of physical effort in the accomplishment of a task. One participant noted that "to reduce risk of over-compensation and associated skill loss and impact on independence and identity, technology could be engineered in a 'reverse training wheels' model (i.e., start with minimal and elder-controlled settings, with escalation of services based on severity of dementia)."

\section{Build in Choice and Control}

Nearly all participants emphasized the need for control to be offered to older adults and their families in the design and use of these technologies. One participant underscored the difficulty with generalizing preferences to the population category "older adults," writing that "responses to a given technology will depend on many personal, social, and situational factors." They continued, "Generalization is difficult because you need to identify which values are important to the older adult. For some it's safety, others privacy, independence, personal control. Preference affects response to and usage of technology." In concert with this important point, participants agreed that designers should adhere to the core design principle of providing personal control options for any system that monitors individual activities. One explained the importance of enabling a pause option: "My prior work found that allowing the older adult to pause whatever the technology is helped with their concerns. They rarely paused it, but being able to was psychologically calming."

An additional specific way to give consumers control is allowing them to delete data and approve data before sharing it in non-emergency situations. One participant suggested setting limits on the capabilities of the technology to remain within the bounds that a person understands and can control. Yet another innovative approach is to use end-user defined function metrics as goals rather than physiological metrics when deciding alert thresholds.

Finally, while it may be apparent to those unfamiliar with familial care dynamics, older adults with dementia should be allowed to stop or cease use of a technology or simply choose not to use it. As one participant suggested, "Alternative options that mean these do not need to be implemented for people/care dyads who just don't want them. People do have different preferences, and they vary, so there should be alternative ways to care for people if they are not comfortable doing these."

\section{Restrictions for Data Security and Unsanctioned Third-Party Use}

Most participants emphasized the need to make mandatory best practices for data security, including for example, two-factor authentication, encryption, HIPAA compliance, and permissions. All data transmitted within and outside of the home can and should be encrypted, according to a number of participants. Some expressed concern over the possibility that data would be used by insurers to deny coverage or increase premiums. Disclosures should indicate that data won't result in higher 
costs. Consumers, they asserted, should have control over their data. Many echoed this participant's suggestion: "Ensure that enrollment is NOT through a primary care or insurance related benefit so that the data collected is the property of the individual and/or family member." Others wrote that data should not be used for marketing or any use other than care provision and that if data are used for research to improve care, consent from individuals should be obtained first. Data should be shared on an opt-in basis. Participants wanted assurances of data privacy and to know how that will be guaranteed, noting that limiting access to data should be carefully controlled.

\section{Require Data Transparency}

Closely related to the issue of data security is that of data transparency. Requiring data transparency means addressing the following questions: Where are the data going? Who can use them? What are the algorithms used to trigger intervention? Are the data being monetized? In addition to transparency about the answers to these questions, devices should actively provide reminders of how they operate, who is controlling them, and how data are being collected and used. The design concept of feedback should be enabled to alert people when monitoring is occurring; this might be in the form of making a noise when it's turning on or using visual feedback like the colored LED ring on Alexa that indicates when the device is listening. Transparency and accessible communication at each of these levels is important to mitigate some of the identified risks.

\section{Enable Informed Consent}

One component of this transparency of data usage is ensuring that consent for using the technology is given by older adults and making sure that consent is fully informed. One participant explained:

Overall, I think for me the most important consideration in cases where there is no control over how the technology was developed, is a solid, ongoing and fully informed consent process, so any way to aid this is valuable: values assessment, maybe multimedia tools to make sure end-users understand what happens to their data/who it's shared with/etc., clear illustrations of possible benefits and harms, etc.

Another participant wrote that while achieving consent may not always be possible, "all of it needs to be with consent." This can present an ethical conflict, as illustrated in this comment about virtual reality: "Although VR might not seem ethically problematic, the degree of immersiveness might be problematic for people with dementia if it is put on them without them having a viable way to say they do not wish to be a part of it." Another wrote that "monitoring related to security (entry and leaving, audio surveillance, video surveillance, etc.) brings with it an increased likelihood of illicit use by unscrupulous persons, which may include family. Elders need to be well counseled in the risks associated with monitoring even if it's for their own good." 
Participants suggested establishing informed consent as a process rather than a one-time event so that people could revisit their use. They recommended repetitive opportunities to try it out, decline to use, and then try again as technologies change and develop. Some acknowledged the possibility that a person living with dementia would not have static preferences as their condition progresses and thus recommended that values assessment be done at earlier stages of disease progression. Conversations that included advanced directives about the use of technologies that monitor and use AI were called for. Finally, participants suggested that when a device has been chosen, people living with dementia could be included in the installation process and onboarding.

Table 5 shows the technologies most commonly selected in response to the following prompt: "Consider which of the below are the most likely to be threatening to values that matter to older adults. Please select six that most warrant a conversation with an older patient when families are deciding about using them in their care."

\section{Proactively Educate Users}

Participants described the need for companies to be responsible for educating consumers about their devices, suggesting that this should be done using language that emphasized person-centered care practices. Participants recommended including disclosures explaining that all systems can fail or have glitches, that oversight and backup provisions are necessary, and that AI is not a substitute for contact with a person living with dementia. User manuals could proactively disclose potential concerns and how to adapt systems, such as reducing frequency of non-critical alerts. A participant noted that it should be acknowledged that "caring" for the technology represents additional work for caregivers and that some of the data gathered may need clinician interpretation before release to the family.

Table 5 Number of participants selecting technologies that most warrant a conversation about potential use

Number of participants out of 21

18 Recording audio in a person's home to respond to emergencies and security threats, monitor socialization, or detect cognitive change and other conditions

16 Video conferencing that would allow a caregiver to turn the webcam on and "enter" the room visually for social connection and visual assessment of person and home

15 Location tracking within the home to detect variation in patterns of activity

13 Location tracking outside of the home to monitor safety and social activity

12 Using an AI virtual agent or socially assistive robot for non-human companionship to ease loneliness and prompt cognitive or physical engagement

11 Detecting movements through the apartment door to confirm return home and monitor outdoor activity frequency or presence of workers, meal delivery, and visitors

11 Remotely monitoring physiological variables for frequent assessment to predict and manage health-related risks 
Another participant offered a suggestion for vendors to help families have conversations and plans for using technology:

A service wrap-around to products that include any or all of the following: examples of how the technology has been used by others, conversation suggestions to support family discussions around the use of the technology, access to human support for the conversation. Human support could take the form of a venderemployed care guide or connection to peer experts in the users' community.

Others recommended educational materials to guide caregiver interactions. For products that have significant potential effect on quality of life, care, routine, or privacy, one suggested mandatory onboarding materials or training that the caregiver should complete before using the product.

\section{Discussion of Implications}

Drawing on the expertise of 21 gerontechnology academic and industry domain experts, we identify which technologies may be the most prevalent in dementia care in five years, along with their individual and shared potential benefits and risks. This article offers a number of unique contributions. These include descriptions of dominant technology categories and a detailed list of primary risks and benefits associated with each (Table 2). We describe the range of options offered to help mitigate the risks and to promote person-centered technology use with this growing population.

While support for people living with dementia and families were top of mind for all participants, these findings suggest important implications for all stakeholders, including systems designers, clinicians, ethicists, researchers, and policy makers. Participants explained that the identified risks specific to each technology (Table 2) and the aggregated risks (Table 3 ) require intervention at multiple levels because they are consequences of choices made at each stage. Some are issues of design that could be mitigated by making different design choices or designing in new options for the user, while others, such as security, may best be addressed through regulatory choices. Intervention should thus begin with systems design and weave into implementation and regulation, following the seven categories described in detail above (summarized in Table 4). Below, we build on the statements provided by participants and discuss further the implications of a subset of their suggestions: employing appropriate design frameworks, enabling control and informed consent, and protecting data and transparency.

\section{Employing Appropriate Design Frameworks}

Many of the concerns raised do need to inform design, and existing frameworksvalue sensitive design, privacy-by-design, and the ethical adoption frameworkshould be incorporated into design processes now. Value sensitive design (VSD) focuses systematically on human values through theory, methods, and practice in the design process (Friedman and Hendry 2019, 3-4). It acknowledges that values 
cannot be easily isolated, because they sit in relationship with other values (e.g., autonomy and identity, privacy and trust) (Friedman and Hendry 2019). A respondent echoed this fact, writing that "human 'values' tend to be mixed up and contradictory." Friedman and Hendry (2019) use the term "value tensions," which, they write, "conveys the idea of values potentially in opposition but allows for solutions that balance each value in relation to the others, such that the adjudication of the tension holds each value intact" (p. 44). This redirects design away from zero-sum thinking. Value tensions can exist internally, between people, and among institutions.

Attention to human values in design is still a novel approach, and its lack of mainstream adoption has negative consequences for products and the people who use them (Davis 2015). If values and potential value tensions were unearthed in the design process, clinicians, care coordinators, and families could be spared the burden of having to navigate products that aren't grounded in values. Value sensitive design has promise to lead to creative accommodations in thoughtfully designed technologies that are made with human values at the center.

The privacy-by-design framework was developed in recognition of the increasing value of data and the urgency to manage them responsibly (Cavoukian 2011). Among its seven foundational principles is making user-centric choices, such as offering strong privacy defaults, transparency, and appropriate notice, and empowering user-friendly options. The privacy-by-design approach is embedded and proactive, anticipating and preventing privacy invasion. It positions privacy as the default such that users do not need to take action to protect it (Cavoukian 2011). It eschews the unnecessary trade-offs of false dichotomies (e.g., privacy vs. security) in favor of accommodating positive-sum interests (Cavoukian and Emam 2010; Cavoukian 2011). Intimate privacy threats have perhaps received greater attention where they occur in intimate partner and parent/child relationships. Schneier and Levy (2020) include elder care in their analysis of privacy threats in intimate relationships. They remind readers, "The fact that intimate information-sharing is widespread and often accepted should not lead us to be unreflective about very real privacy threats within intimate relationships. Rather, it makes it all the more important to consider how intimate privacy threats occur, when they are unwelcome, and how to reason about them conceptually" (Schneier and Levy 2020, pp. 2-3). Threats to privacy was among the most commonly endorsed risks of using these dementia care technologies, which indicates that there is significant work to be done yet to protect the privacy of individuals and families.

Finally, the ethical adoption framework is grounded in bioethics and was developed specifically for technology for dementia care. It addresses not just the design but also the integration of design, development, deployment, and use (Robillard et al. 2018). The framework includes 18 recommendations based on five pillars that are supported by empirical evidence: inclusive participatory design, emotional alignment, adoption modelling, ethical standards assessment, and education and training (Robillard et al. 2018). This framework targets multiple phases of technology trajectory, including early design, delivery, adoption promotion, ethical standards review, user testing, launch, and continued use (Robillard et al. 2018). 


\section{Enabling Control and Informed Consent}

Enabling informed consent and proactively educating users were prioritized needs, as were built-in options for choice and control. Participants cautioned against generalizing ideas of how people prioritize implicated values, stressing the need to understand that this will vary by person. This work and others (Mulvenna et al. 2017; Berridge 2018; Wangmo et al. 2019; Berridge and Wetle 2019) suggest that we need to further investigate how to support personalized patient and family decision-making about these forms of technologies used in dementia care with attention to alignment with participants' cultural values (Berridge et al. 2019).

To a great extent, those technologies participants rated most likely to cause family conflict were also predicted to be most prevalent in five years in dementia care. Because these are predicted to be the most prevalent soon, the need to learn how to ensure that families are well counseled about their risks may be the most immediate. The five deemed most likely to call for conversations and predicted to be among the most prevalent were video conferencing that allows a caregiver to turn the webcam on and "enter" the room; location tracking outside of the home; location tracking within the home; detecting movements through an apartment door; and remotely monitoring physiological variables. The technology most likely to cause conflictrecording audio-was not expected to be as prevalent as the other options, though some of those do include audio recording capacity.

Using AI conversational agents was ranked fifth for potential to cause conflict and seventh for prevalence in five years. A number of respondents used the word "problematic" in reference to using AI for companionship and social interaction. The primary ethical issues with artificial companions discussed in the literature were also noted by participants. These include the likelihood of deception, privacy invasion through monitoring and tracking, and the possibility that such devices could ease or contribute to social isolation (Robillard et al. 2020; Wangmo et al. 2019). The potential for artificial companions to ease loneliness is actively designed for and promoted, yet the regulatory landscape is weak (Portacolone et al. 2020), uninformed by input from older adults and advocates (Robillard et al. 2020). The current study's findings align with the recommendation that resources are needed to examine the ethical aspects and implications of such use-research that meaningfully engages older adults and caregivers - and to put protective policies in place prior to broader implementation (Robillard et al. 2019).

Strikingly little research has focused on the convergence of policy and ethical issues in dementia care technology use (Robillard et al. 2019). A recent policy analysis describes state Medicaid waiver programs' difficulty regulating geo-tracking, activity sensors, and web-cameras that some are allowing and paying for. Program managers report struggling to understand which circumstances warrant use of these devices and how to support ethical decision making for beneficiaries with cognitive impairment (Berridge 2018). Another ethics analysis of 23 policy documents from four Alzhiemer's Associations in four countries found that they prioritize benefits of technologies while inadequately discussing potential harms. The authors note "a lack of formal guidance and governing for both existing and emerging technologies" (Robillard et al. 2019). The current study further underscores why-absent such 
guidance and regulation-families, front line staff, and policy makers will continue to struggle with ethical and low-risk use of dementia care technology.

\section{Protecting Data and Transparency}

Data security and transparency were also strong themes. These data collected about older adults are potentially financially valuable and present risks to personal privacy. Consumer protections, enacted through policy or law, that mandate best practices such as two-factor authentication, encryption, HIPAA compliance, data-use transparency, and consumer controls (e.g., clearly-communicated opt-in rather than optout options) will be essential.

Data transferred from a range of devices_-particularly those that collect physiological data-might be used by insurance companies to restrict access or increase costs of care. Preventing direct relationships between health insurers and device companies was recommended. Regulation appears overdue. Health insurance companies such as Aetna are launching products that use data collected by personal data devices - in this case, a collaboration with Apple to launch an app using Apple Watch data (Shieber 2019). The public also has good reason not to trust that its data are safe from unsanctioned use, as shown recently by the secret transfer by Ascension of nearly 50 million consumers' medical data to Google (Project Nightingale) (Pilkington 2019). Study participants felt that family caregivers should be relieved of the burden of such risks. This will require significant policy work and new mechanisms for regulators to keep up with device changes (Duggal et al. 2018).

The specific recommendations offered here are relevant to designers, clinicians, ethicists, researchers, and policy makers. It is clear that there is no catch-all solution and that intentional choices need to be made to address these risks across design and implementation.

\section{Limitations}

A greater proportion of academic researchers than industry representatives participated in this Delphi process; however, many of them were also engaged in product development as part of their research. The majority of participants did not have specific expertise in ethics, and participants were not asked to comment on ethical issues; rather, questions were framed in terms of potential benefits and risks. While ethical issues were raised, this study does not and was not intended to represent a thorough review of ethical issues. For explicit focus on ethics, see for example, Wangmo, et al. (2019) and Vallor (2016). Still needed is the engagement of experts in $\mathrm{AI}$ and other applications who are not working in gerontechnology. The methods of convenience and snowball sampling to identify prospective participants may limit the representativeness of our sample.

The focus of this study was on technologies used in dementia care by families. While some insights are broadly applicable, specific contexts of nursing homes or other long-term care residential settings were not explored. Further, this study does not address the problem of the exclusion of people living with dementia in research 
(Taylor et al. 2012) that is also a limitation in gerontechnology. More studies that include people living with dementia are needed if we are to understand the full scope of potential risks, benefits, risk mitigation efforts, and the ways digital technologies open and close ways of being and experiencing daily life.

\section{Appendix: List of participants}

Walter R. Boot, Professor of Psychology, Florida State University.

Christine Cassel, Presidential Chair, University of California at San Francisco.

Neil Charness, William G. Chase Professor of Psychology and Director, Institute for Successful Longevity, Department of Psychology, Florida State University.

R. Scott Collins, Chief Executive Officer, Link-age.

Kay Connelly, Professor and Associate Dean for Research in the School of Informatics, Computing, and Engineering, Indiana University.

James Fogarty, Professor, Computer Science and Engineering, University of Washington.

Joseph Gaugler, Professor and Robert L. Kane Endowed Chair in Long-Term Care and Aging, School of Public Health, University of Minnesota.

William Kearns, Associate Professor, Meritorious (Retired) Rehabilitation and Mental Health Counseling, Department of Child and Family Studies, University of South Florida, President, International Society for Gerontechnology.

Amanda Lazar, Assistant Professor, College of Information Studies, University of Maryland.

Diane Mahoney, Professor Emerita MGH Institute for Health Professions, School of Nursing.

Barbara Marshall, Professor of Sociology, Trent University.

Debra Oliver, Professor and Paul Revare, MD, Family Professor of Family Medicine, University of Missouri.

Laurie Orlov, Tech industry veteran and founder, Aging in Place Technology Watch.

Carla Perissinotto, Associate Professor in the Division of Geriatrics and Associate Chief for Geriatrics Clinical Programs, University of California San Francisco.

Marilyn Rantz, Curators' Professor Emerita, School of Nursing, University of Missouri, Executive Director, Aging In Place Project and Sinclair Home Care.

Julie Robillard, Assistant Professor of Neurology, University of British Columbia and Associate Director of Neuroethics Canada.

Brian Shulman, Director, Business Development, Intuition Robotics.

Andrew Sixsmith, Professor in the Department of Gerontology, Simon Fraser University, Director of the Science and Technology for Aging Research (STAR) Institute, Scientific Director of AGE-WELL NCE, Canada's AgingTech network.

Nancy Vuckovic, Director of Experience Design, Cambia Health Solutions.

Erin Washington, Co-Founder and Chief Product Officer, Embodied Labs.

Jutta Williams, Sr. Technical Program Manager, Facebook. 
Funding This work was supported by the National Institutes of Health [K01AG062681 to C.B., P30-AG008017, P30-AG024978, and U2CAG054397 to J.K.]

Open Access This article is licensed under a Creative Commons Attribution 4.0 International License, which permits use, sharing, adaptation, distribution and reproduction in any medium or format, as long as you give appropriate credit to the original author(s) and the source, provide a link to the Creative Commons licence, and indicate if changes were made. The images or other third party material in this article are included in the article's Creative Commons licence, unless indicated otherwise in a credit line to the material. If material is not included in the article's Creative Commons licence and your intended use is not permitted by statutory regulation or exceeds the permitted use, you will need to obtain permission directly from the copyright holder. To view a copy of this licence, visit http://creativecommons.org/licen ses/by/4.0/.

\section{References}

AARP and National Alliance for Caregiving. (2020). Caregiving in the United States 2020. Washington, DC: AARP. https://doi.org/10.26419/ppi.00103.001.

Akins, R. B., Tolson, H., \& Cole, B. R. (2005). Stability of response characteristics of a Delphi panel: Application of bootstrap data expansion. BMC Medical Research Methodology., 5, 37. https://doi. org/10.1186/1471-2288-5-37.

Berridge, C. (2016). Breathing room in monitored space: The impact of passive monitoring technology on privacy in independent living. The Gerontologist, 56(5), 807-816.

Berridge, C. (2018). Medicaid becomes the first third-party payer to cover passive remote monitoring for home care: Policy analysis. Journal of Medical Internet Research, 20(2), e66. https://doi.org/10. 2196/jmir.9650.

Berridge, C., Chan, K. T., \& Choi, Y. (2019). Sensor-based passive remote monitoring and discordant values: Qualitative study of the experiences of low-income immigrant elders in the United States. JMIR mHealth and uHealth, 7(3), e11516. https://doi.org/10.2196/11516.

Berridge, C., \& Wetle, T. F. (2019). Why older adults and their children disagree about in-home surveillance technology, sensors, and tracking. The Gerontologist. https://doi.org/10.1093/geront/gnz068.

Boise, L., Wild, K., Mattek, N., Ruhl, M., Dodge, H., \& Kaye, J. (2013). Willingness of older adults to share data and privacy concerns after exposure to unobtrusive in-home monitoring. Gerontechnology, 11, 428-435.

Cavoukian, A. (2011). Privacy by design-Strong privacy protection-Now, and well into the future. In A report on the state of $P b D$ to the 33rd international conference of data protection and privacy commissioners. https://www.ipc.on.ca/wp-content/uploads/Resources/PbDReport.pdf.

Cavoukian, A., \& Emam, K. E. (2010). A positive-sum paradigm in action in the health sector. Whitepaper, Office of the Information and Privacy Commissioner of Ontario. https://www.ipc.on.ca/wpcontent/uploads/2010/03/A-Positive-Sum-Paradigm-in-Action-in-the-Health-Sector.pdf.

Choi, Y., Lazar, A., Demiris, G., \& Thompson, H. (2019). Emerging smart home technologies to facilitate engaging with aging. Journal of Gerontological Nursing, 45, 41-48. https://doi.org/10.3928/ 00989134-20191105-06.

Clarke, V., Braun, V., Terry, G., \& Hayfield, N. (2019). Thematic analysis. In P. Liamputtong (Ed.), Handbook of research methods in health and social sciences (pp. 843-860). Singapore: Springer.

Czaja, S. J., Boot, W. R., Charness, N., \& Rogers, W. A. (2019). Designing for older adults: Principles and creative human factors approaches ( $3 \mathrm{rd}$ ed.). Boca Raton: Taylor \& Francis.

Davis, J. (2015). Value sensitive design: Applications, adaptations, and critiques. In J. Van den Hoven, P. E. Vermaas, \& I. Van de Poel (Eds.), Handbook of ethics, values, and technological design: SOURCES, theory, values and application domains (pp. 125-140). Dordrecht: Springer.

Duggal, R., Brindle, I., \& Bagenal, J. (2018). Digital healthcare: Regulating the revolution. BMJ, 15(360), k6. https://doi.org/10.1136/bmj.k6.

Friedman, B., \& Hendry, D. G. (2019). Value sensitive design. Cambridge, MA: MIT Press.

Hsu, C. C., \& Sandford, B. A. (2007). The Delphi technique: Making sense of consensus practical assessment. Practical Assessment Research \& Evaluation, 12, 1-10. http://pareonline.net/getvn.asp?v= $12 \& n=10$. Accessed 2 April 2018. 
Ienca, M., Wangmo, T., Jotterand, F., Kressig, R. W., \& Elger, B. (2018). Ethical Design Of Intelligent Assistive Technologies for dementia: A descriptive review. Science and Engineering Ethics, 24(4), 1035-1055.

Kenner, A. M. (2008). Securing the elderly body: Dementia, surveillance, and the politics of "aging in place." Surveillance \& Society Journal, 5(3), 252-269.

Lindeman, D. A., Kim, K. K., Gladstone, C., \& Apesoa-Varano, E. C. (2020). Technology and caregiving: Emerging interventions and directions for research. The Gerontologist, 60(Sup 1), S41-S49. https://doi.org/10.1093/geront/gnz178.

Meiland, F., Innes, A., Mountain, G., et al. (2017). Technologies to support community-dwelling persons with dementia: A position paper on issues regarding development, usability, effectiveness and cost-effectiveness, deployment, and ethics. JMIR Rehabilitation and Assistive Technologies, 4(1), e1. https://doi.org/10.2196/rehab.6376.

Mokkink, L. B., Terwee, C. B., Patrick, D. L., Alonso, J., Stratford, P. W., Knol, D. L., et al. (2010). The COSMIN checklist for assessing the methodological quality of studies on measurement properties of health status measurement instruments: An international Delphi study. Quality of Life Research, 19(4), 539-549. https://doi.org/10.1007/s11136-010-9606-8 (Epub 2010 Feb 19).

Mulvenna, M., Hutton, A., Coates, V., Martin, S., Todd, S., \& Bond, R. (2017). Views of caregivers on the ethics of assistive technology used for home surveillance of people living with dementia. Neuroethics, 10(2), 255-266.

Novitzky, P., Smeaton, A. F., Chen, C., Irving, K., Jacquemard, T., O’Brolcháin, F., et al. (2015). A review of contemporary work on the ethics of ambient assisted living technologies for people with dementia. Science and Engineering Ethics, 21(3), 707-765.

Orlov, L. (2019). Aging in place technology watch. Retrieved from https://www.ageinplacetech.com/blog/ voice-health-and-well-being-launching-new-report-2020.

Piau, A., Wild, K., Mattek, N., \& Kaye, J. (2019). Current state of digital biomarker technologies for reallife, home-based monitoring of cognitive function for mild cognitive impairment to mild Alzheimer disease and implications for clinical care: Systematic review. Journal of Medical Internet Research, 21(8), e12785. https://doi.org/10.2196/12785.

Pilkington, D. (2019). Google's secret cache of medical data includes names and full details of millionsWhistleblower. The Guardian. https://www.theguardian.com/technology/2019/nov/12/google-medic al-data-project-nightingale-secret-transfer-us-health-information.

Pols, J. (2017). Good relations with technology: Empirical ethics and aesthetics in care. Nursing Philosophy, 18(1), e12154. https://doi.org/10.1111/nup.12154.

Portacolone, E., Halpern, J., Luxenberg, J., Harrison, K. L., \& Covinsky, K. E. (2020). Ethical issues raised by the introduction of artificial companions to older adults with cognitive impairment: A call for interdisciplinary collaborations. Journal of Alzheimer's Disease, 76, 445-455.

Robillard, J. M., Cleland, I., Hoey, J., \& Nugent, C. (2018). Ethical adoption: A new imperative in the development of technology for dementia. Alzheimer's \& Dementia, 14(9), 1104-1113.

Robillard, J. M., Goldmanc, I. P., Prescottd, T. J., \& Michaud, F. (2020). Addressing the ethics of telepresence applications through end-user engagement. Journal of Alzheimer's Disease, 76, 457-460.

Robillard, J. M., Wu, J. M., Feng, T. L., \& Tam, M. T. (2019). Prioritizing benefits: A content analysis of the ethics in dementia technology policies. Journal of Alzheimer's Disease, 69(4), 897-904. https:// doi.org/10.3233/JAD-180938.

Sánchez, V., Taylor, I., \& Bing-Jonsson, P. C. (2017). Ethics of smart house welfare technology for older adults: A systematic literature review. International Journal of Technology Assessment in Health Care, 33(6), 691-699. https://doi.org/10.1017/S0266462317000964.

Schneier, B., \& Levy, K. (2020). Privacy threats in intimate relationships. Journal of Cybersecurity. https://doi.org/10.1093/cybsec/tyaa006.

Seelye, A., Leese, M., Dorociak, K., Bouranis, N., Mattek, N., Sharma, N., et al. (2020). In-home sensor monitoring to detect mild cognitive impairment in aging military veterans: Preliminary data on methods and feasibility. Journal of Medical Internet Research. https://doi.org/10.2196/16371.

Shieber, J. (2019). Apple partners with Aetna to launch health app leveraging Apple Watch data. TechCrunch. https://techcrunch.com/2019/01/29/apple-partners-with-aetna-to-launch-health-app-lever aging-apple-watch-data.

Sinha, I. P., Smyth, R. L., \& Williamson, P. R. (2011). Using the Delphi technique to determine which outcomes to measure in clinical trials: Recommendations for the future based on a systematic review of existing studies. PLoS Medicine, 8(1), e1000393. https://doi.org/10.1371/journal.pmed.1000393. 
Taylor, J. S., DeMers, S. M., Vig, E. K., \& Borson, S. (2012). The disappearing subject: Exclusion of people with cognitive impairment and dementia from geriatrics research. Journal of the American Geriatrics Society, 60(3), 413-419. https://doi.org/10.1111/j.1532-5415.2011.03847.x.

Vallor, S. (2016). Technology and the virtues: A philosophical guide to a future worth wanting. New York, NY: Oxford University Press.

Walker, A. M., \& Selfe, J. (1996). The Delphi technique: A useful tool for the allied health researcher. British Journal of Therapy and Rehabilitation, 3, 677-680.

Wangmo, T., Lipps, M., Kressig, R. W., et al. (2019). Ethical concerns with the use of intelligent assistive technology: Findings from a qualitative study with professional stakeholders. BMC Medical Ethics, 20, 98.

Ziglio, E. (1996). The Delphi method and its contribution to decision making. In M. Adler \& E. Ziglio (Eds.), Gazing into the Oracle: The Delphi method and its application to social policy and public health (pp. 3-33). Bristol, PA: Jessica Kingsley Publishers.

Publisher's Note Springer Nature remains neutral with regard to jurisdictional claims in published maps and institutional affiliations.

\section{Authors and Affiliations}

\section{Clara Berridge $^{1}$ D $\cdot$ George Demiris $^{2} \cdot$ Jeffrey Kaye ${ }^{3}$}

George Demiris

gdemiris@upenn.edu

Jeffrey Kaye

kaye@ohsu.edu

1 School of Social Work, University of Washington, Seattle, WA, USA

2 School of Nursing and Perelman School of Medicine, University of Pennsylvania, Philadelphia, PA, USA

3 School of Medicine, Oregon Health and Science University, Portland, OR, USA 